\title{
Mechanism Underlying Induction of Hyperglycemia in Rats by Single Administration of Olanzapine
}

\author{
Masashi Nagata, ${ }^{a}$ Mayumi Nakajima, ${ }^{a, b}$ Yasuyoshi Ishiwata, ${ }^{a}$ Yutaka Takahashi, ${ }^{a}$ \\ Hiromitsu Takahashi, ${ }^{a}$ Kenichi Negishi, ${ }^{b}$ and Masato Yasuhara*c \\ ${ }^{a}$ Department of Pharmacy, Medical Hospital, Tokyo Medical and Dental University; 1-5-45 Yushima, Bunkyo- \\ ku, Tokyo 113-8519, Japan: ${ }^{b}$ Faculty of Pharmaceutical Sciences, Tokyo University of Science; 2641 Yamazaki, \\ Noda, Chiba 278-8510, Japan: and ${ }^{c}$ Department of Pharmacokinetics and Pharmacodynamics, Graduate School of \\ Medical and Dental Sciences, Tokyo Medical and Dental University; 1-5-45 Yushima, Bunkyo-ku, Tokyo 113-8519, \\ Japan.
}

Received October 28, 2015; accepted January 7, 2016

Acute administration of olanzapine rapidly elevates blood glucose levels. However, the mechanism underlying the rapid development of hyperglycemia with the administration of olanzapine remains unclear. The aim of the present study was to clarify the mechanism underlying olanzapine-induced acute hyperglycemia. Male Wistar rats received an intravenous infusion of saline (control) or olanzapine $2.5,5$, or $10 \mathrm{mg} / \mathrm{kg}$. Blood samples were obtained periodically after olanzapine infusion to determine serum concentrations of glucose, olanzapine, and several endogenous substances. In a separate experiment, rats received an intravenous injection of propranolol $(2 \mathrm{mg} / \mathrm{kg}) 30 \mathrm{~min}$ before infusion of olanzapine $(10 \mathrm{mg} / \mathrm{kg})$. The intravenous infusion of olanzapine induced dose-dependent increases in the serum concentrations of glucose, epinephrine, and insulin. Pretreatment with propranolol suppressed olanzapine-induced elevations in the serum concentration of glucose, but did not affect the serum concentration of olanzapine or olanzapine-induced increase in the serum concentration of epinephrine. Although the serum concentration of corticosterone increased after administration of olanzapine, no significant differences were observed among the olanzapine dose groups. Furthermore, administration of olanzapine did not affect the serum concentration of glucagon or histamine. We developed a pharmacokinetic-pharmacodynamic model assuming that the olanzapine-induced secretion of epinephrine leads to elevated serum glucose concentrations. This model appeared to satisfactorily characterize olanzapine-induced hyperglycemia. In conclusion, a single intravenous dose of olanzapine dosedependently increased the serum concentration of glucose in rats, and epinephrine plays a role in olanzapineinduced acute hyperglycemia.

Key words olanzapine; hyperglycemia; epinephrine; rat

Olanzapine, an atypical antipsychotic drug, is considered to effectively relieve various psychiatric symptoms such as positive symptoms, negative symptoms, and dementia due to its broad and potent inhibitory activity at multiple receptors such as dopamine, serotonin, $\alpha_{1}$-adrenergic, and histamine $\mathrm{H}_{1}$ receptors. ${ }^{1)}$ Due to the lower incidence of extrapyramidal side effects associated with olanzapine, it is currently used as one of the first-line drugs to treat schizophrenia. ${ }^{2}$ However, a previous study reported the development of diabetic ketoacidosis and coma due to hyperglycemia following the administration of olanzapine. ${ }^{3)}$ Therefore, Emergency Safety Information on these side effects was issued in Japan (2002), and olanzapine became contraindicated for patients with diabetes mellitus. Furthermore, warnings related to blood glucose monitoring in all patients treated with olanzapine have been added to the labeling of olanzapine.

Previous studies have attempted to elucidate the mechanism responsible for the induction of hyperglycemia with the chronic administration of olanzapine. Olanzapine treatments may be associated with increased appetite and concomitant weight gain and obesity, which, in turn, may induce hyperglycemia and type 2 diabetes mellitus. ${ }^{4)}$ Olanzapine treatments have recently been suggested to directly affect insulin resistance and glucose homeostasis independent of weight gain, increases in food intake, and psychiatric diseases. ${ }^{4,5}$
On the other hand, the acute administration of olanzapine is known to rapidly elevate blood glucose levels. Kohen et al. reported that an 89-year-old man without diabetes developed hyperglycemia $3 \mathrm{~d}$ after the initiation of olanzapine. $\left.{ }^{6}\right)$ This hyperglycemia resolved when the administration of olanzapine was stopped and then recurred $2 \mathrm{~d}$ after a rechallenge with olanzapine. Mizukami et al. described a 22-year-old woman without diabetes who developed hyperglycemia (her glucose concentration exceeded the detection limits) $4 \mathrm{~h}$ after the first administration of olanzapine. ${ }^{7)}$ Regarding the mechanism responsible for the induction of acute hyperglycemia by olanzapine, Ikegami et al. recently showed that olanzapine activates adenosine 5'-monophosphate-activated protein kinase (AMPK) in the hypothalamus, which increases the hepatic production of glucose via the sympathetic nervous system. $\left.{ }^{8}\right)$ They also suggested that olanzapine activates hypothalamic AMPK by antagonizing histamine $\mathrm{H}_{1}$, dopamine $\mathrm{D}_{2}$, and $\alpha_{1}$-adrenergic receptors. ${ }^{9)}$ However, it has not yet been determined whether olanzapine affects several endogenous substances associated with glucose homeostasis thorough the activation of AMPK.

In the present study, we investigated the effects of a single intravenous administration of olanzapine on the serum concentrations of glucose and several endogenous substances such as epinephrine, corticosterone, and glucagon in rats. 


\section{MATERIALS AND METHODS}

Animals Male Wistar rats (Japan SLC, Hamamatsu, Japan) weighing 180-230g were used. Rats were housed in a controlled environment and fasted overnight before the experiment. In in vivo studies, rats had indwelling cannulas implanted in the left carotid artery and jugular vein under pentobarbital anesthesia $(30 \mathrm{mg} / \mathrm{kg}$, intraperitoneally) for blood sampling and intravenous injections, respectively, and were fasted during the experiment. Animal experiments were performed in accordance with the Guidelines for Animal Experiments of Tokyo Medical and Dental University.

Materials Olanzapine was purchased from Wako Pure Chemical Industries, Ltd. (Osaka, Japan). Regarding the intravenous infusion, $10 \mathrm{mg}$ of olanzapine was dissolved in $1.2 \mathrm{~mL}$ of $0.1 \mathrm{~m}$ hydrochloric acid and the solution was adjusted to 0.5 , 1 , and $2 \mathrm{mg} / \mathrm{mL}$ with saline. (士)-Propranolol hydrochloride was purchased from Wako Pure Chemical Industries, Ltd. and dissolved in saline $(0.8 \mathrm{mg} / \mathrm{mL})$. Caffeine (as an internal standard for the assay of olanzapine), $(R)-(-)$-epinephrine, histamine dihydrochloride, and betamethasone (as an internal standard for the assay of corticosterone) were purchased from Wako Pure Chemical Industries, Ltd. Corticosterone and potassium ferricyanide(III) was purchased from Sigma-Aldrich (St. Louis, MO, U.S.A.). $o$-Phthalaldehyde was purchased from Nacalai Tesque Inc. (Kyoto, Japan). $(1 R, 2 R)-(+)-1,2-D i p h e n y l-$ ethylenediamine and methacholine chloride were purchased from Tokyo Chemical Industry Co., Ltd. (Tokyo, Japan). All chemicals were of analytical grade.

Effects of the Single Intravenous Administration of Olanzapine on Serum Concentrations of Glucose and Several Endogenous Substances in Rats In order to limit the volume of blood sampling, our experiment was performed on 3 sets of rats. In the first set of rats, olanzapine at 2.5, 5, or $10 \mathrm{mg} / \mathrm{kg}$ was intravenously infused for $5 \mathrm{~min}$ to rats $1 \mathrm{~h}$ after awakening from anesthesia. Blood samples $(500 \mu \mathrm{L})$ were obtained $0,15,30,60,180$, and $360 \mathrm{~min}$ after the end of the olanzapine infusion to determine the serum concentrations of glucose, glucagon, insulin, and corticosterone. An equivalent volume of normal saline was infused into control rats.

In the second set of rats, olanzapine at $2.5,5$, or $10 \mathrm{mg} / \mathrm{kg}$ was intravenously infused for $5 \mathrm{~min}$ to rats $1 \mathrm{~h}$ after awakening from anesthesia. Blood samples $(700 \mu \mathrm{L})$ were obtained 0 , $15,30,60,180$, and $360 \mathrm{~min}$ after the end of the olanzapine infusion to determine the serum concentrations of glucose, epinephrine, and olanzapine.

In the third set of rats, olanzapine at 2.5 or $5 \mathrm{mg} / \mathrm{kg}$ was intravenously infused for $5 \mathrm{~min}$ to rats $1 \mathrm{~h}$ after awakening from anesthesia. Blood samples $(1.2 \mathrm{~mL})$ were obtained 0,15 , and $60 \mathrm{~min}$ after the end of the olanzapine infusion to determine the serum concentrations of glucose and histamine.

Effects of Propranolol on Olanzapine-Induced Increases in the Serum Concentration of Glucose in Rats Rats were divided into three groups: propranolol/olanzapine, propranolol/ saline, and saline/olanzapine. Propranolol $(2 \mathrm{mg} / \mathrm{kg})$ or saline was injected intravenously $30 \mathrm{~min}$ after awakening from anesthesia. Thirty minutes after the propranolol/saline injection, olanzapine $(10 \mathrm{mg} / \mathrm{kg})$ or saline was intravenously infused for 5 min. Blood samples $(700 \mu \mathrm{L})$ were obtained $0,15,30,60$, 180 , and $360 \mathrm{~min}$ after the end of the olanzapine/saline infusion to determine the serum concentrations of glucose, epinephrine, and olanzapine.

Analytical Methods The serum concentration of glucose was determined by the glucose oxidase method using Glucose CII-test Wako (Wako Pure Chemical Industries, Ltd.). Serum concentrations of immunoreactive insulin and glucagon were

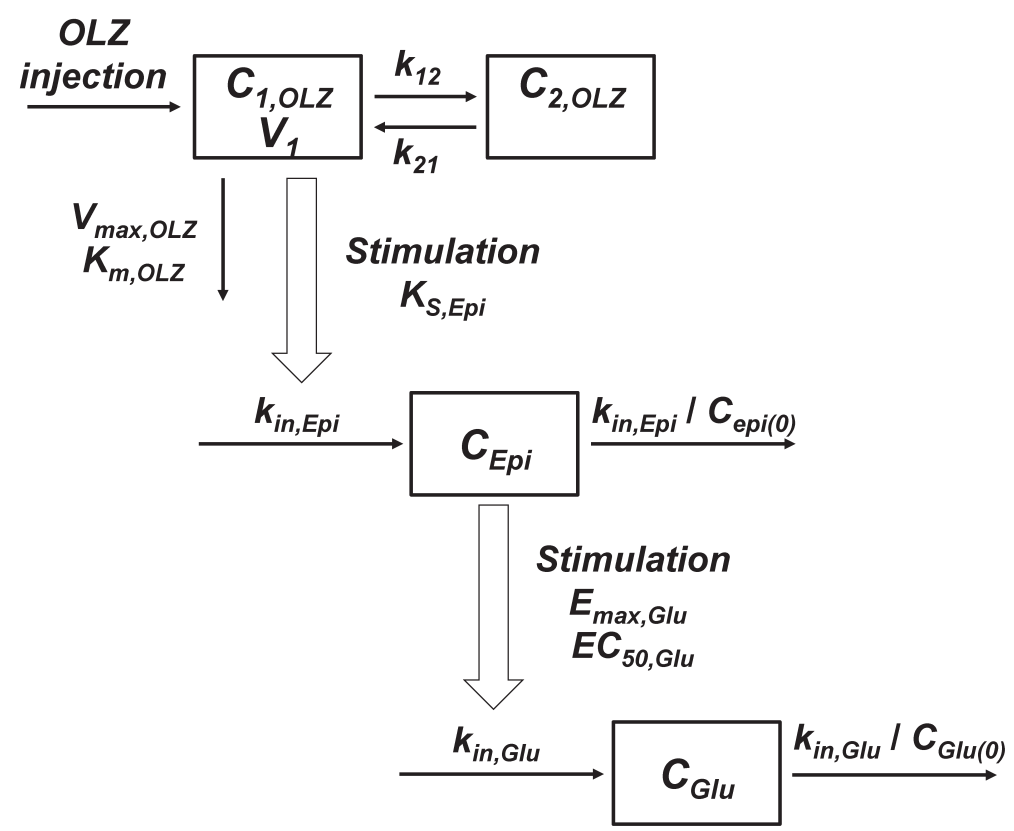

Fig. 1. Schematic Representation of the Pharmacokinetic-Pharmacodynamic Model of Olanzapine-Induced Hyperglycemia

$C_{1, \text { oLZ }}$, the serum concentration of olanzapine in the central compartment; $C_{2, \text { oLZ }}$, the serum concentration of olanzapine in the peripheral compartment; $V_{1}$, the distribution volume of olanzapine in the central compartment; $k_{12}$, the first order transfer rate constant for a drug moving from the central to the peripheral compartment; $k_{21}$, the first order transfer rate constant for a drug moving from the peripheral to the central compartment; $V_{\text {max.olz }}$, the maximum velocity of the elimination of olanzapine;

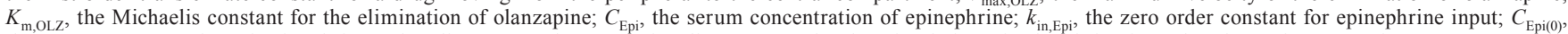
the serum concentration of epinephrine at baseline; $K_{\mathrm{S} \text { Ep }}$, the proportionality constant for the stimulation of epinephrine input by olanzapine; $C_{\mathrm{G} \text { II }}$ the serum concentration of glucose; $k_{\text {in,Glu }}$, the zero order constant for glucose input; $C_{\mathrm{Glu}(0)}$, the serum concentration of glucose at baseline; $E_{\mathrm{max} \text { Glu }}$ the maximum effect for the stimulation of glucose input by epinephrine; $E C_{50, \mathrm{Glu}}$, the epinephrine concentration corresponding to $50 \%$ of the maximum effect for the stimulation of glucose input by epinephrine. 

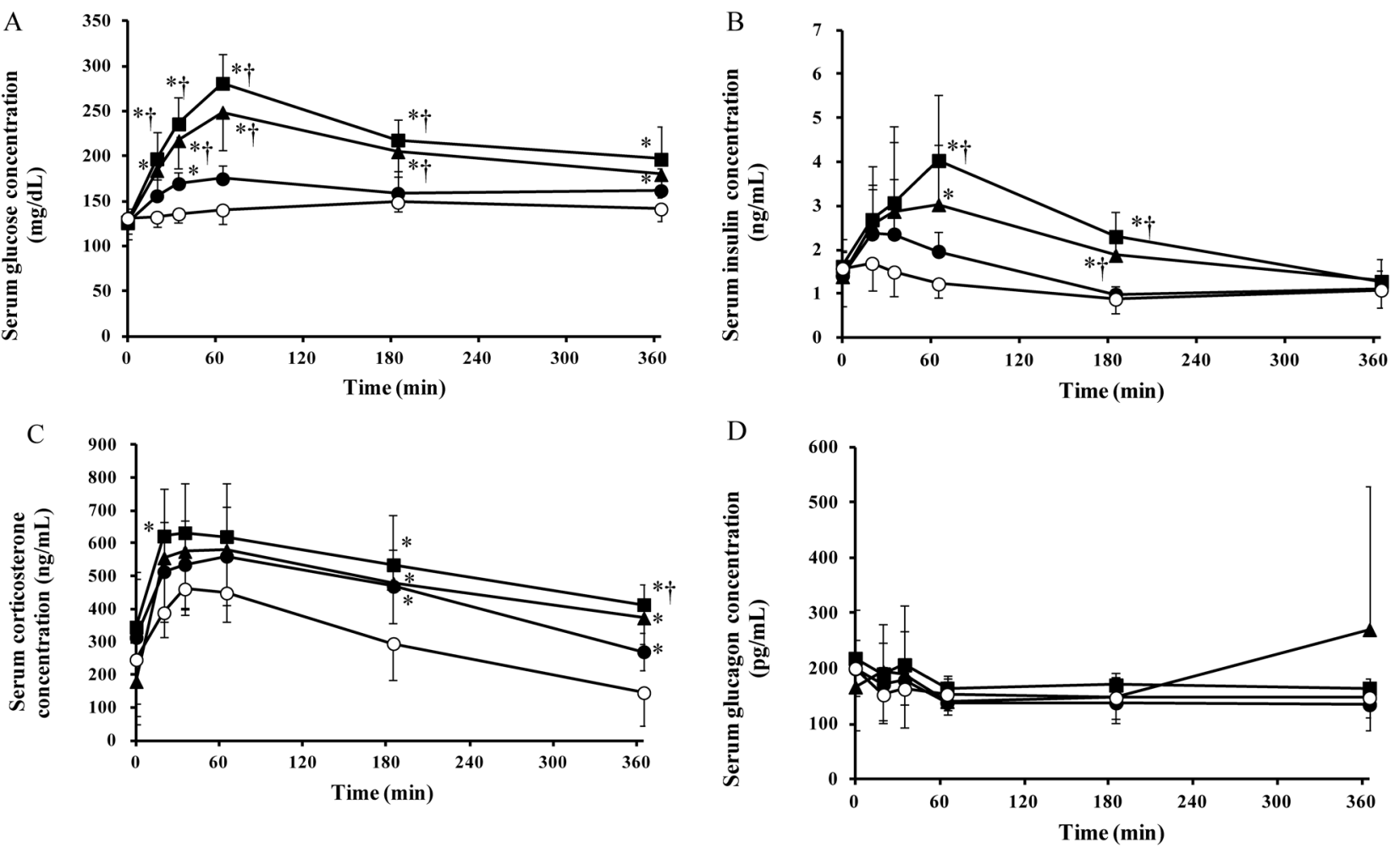

Fig. 2. Serum Concentration-Time Profiles of Glucose (A), Insulin (B), Corticosterone (C), and Glucagon (D) after the Intravenous Infusion of Saline ( $($, as Control, $n=8)$, Olanzapine at $2.5 \mathrm{mg} / \mathrm{kg}(\boldsymbol{\bullet}, n=7)$, Olanzapine at $5 \mathrm{mg} / \mathrm{kg}(\boldsymbol{\Lambda}, n=7)$, or Olanzapine at $10 \mathrm{mg} / \mathrm{kg}(\mathbf{\square}, n=7) \mathrm{in} \mathrm{Rats}$

Each point represents the mean \pm S.D. ${ }^{*} p<0.05$ vs. the control group and ${ }^{\dagger} p<0.05 v s$. olanzapine $2.5 \mathrm{mg} / \mathrm{kg}$ (the Tukey-Kramer test).

determined by an enzyme immunoassay using the Rat Insulin ELISA KIT (TMB) (Shibayagi, Gunma, Japan) and Rat Glucagon ELISA Kit Wako (Wako Pure Chemical Industries, Ltd.), respectively.

Serum concentrations of corticosterone, ${ }^{10)}$ olanzapine, ${ }^{11)}$ epinephrine, ${ }^{12)}$ and histamine ${ }^{13)}$ were determined by HPLC as previously described with slight modifications.

The HPLC apparatus was a LC-10A (Shimadzu Co., Kyoto, Japan) equipped with an ultraviolet spectrometer (SPD-10A, Shimadzu Co.) and spectrofluorometer (RF-550A, Shimadzu Co.). The column was TSK-gel ODS- $80^{\mathrm{TM}}(5 \mu \mathrm{m}, 4.6 \mathrm{~mm}$ i.d. $\times 15 \mathrm{~cm}$, TOSOH, Japan) and was kept at $40^{\circ} \mathrm{C}$.

Pharmacokinetic-Pharmacodynamic Analysis Since the infusion time of olanzapine $(5 \mathrm{~min})$ was shorter than the disposition half-life $(2.5 \mathrm{~h}),{ }^{14)}$ a pharmacokinetic-pharmacodynamic analysis was performed using an intravenous bolus administration model. The pharmacokinetic and pharmacodynamic model for olanzapine-induced hyperglycemia is shown in Fig. 1. Since the area under the concentration-time curve of olanzapine showed a nonlinear increase as the increase of dose from 2.5 to $10 \mathrm{mg} / \mathrm{kg}$, the time profiles of serum olanzapine concentrations were fit to a two-compartment model with Michaelis-Menten elimination kinetics from the central compartment. The differential equations describing the model in Fig. 1 were:

$$
\begin{aligned}
& \frac{\mathrm{d} C_{1, \mathrm{OLZ}}}{\mathrm{d} t}=k_{12} \cdot\left(C_{2, \mathrm{OLZ}}-C_{1, \mathrm{OLZ}}\right)-\frac{V_{\mathrm{max}, \mathrm{OLZ}}}{\left(K_{\mathrm{m}, \mathrm{OLZ}}+C_{1, \mathrm{OLZ}}\right)} \cdot \frac{C_{1, \mathrm{OLZ}}}{V_{1}} \\
& \frac{\mathrm{d} C_{2, \mathrm{OLZ}}}{\mathrm{d} t}=k_{21} \cdot\left(C_{1, \mathrm{OLZ}}-C_{2, \mathrm{OLZ}}\right)
\end{aligned}
$$

where $C_{1, \mathrm{OLZ}}, C_{2, \mathrm{OLZ}}, V_{1}, k_{12}, k_{21}, V_{\text {max }, \mathrm{OLZ}}$, and $K_{\mathrm{m}, \mathrm{OLZ}}$ represent the serum concentration of olanzapine in the central compartment, the serum concentration of olanzapine in the peripheral compartment, the distribution volume of olanzapine in the central compartment, first order transfer rate constant for a drug moving from the central to the peripheral compartment, first order transfer rate constant for a drug moving from the peripheral to the central compartment, the maximum velocity of the elimination of olanzapine, and the Michaelis constant for the elimination of olanzapine. The indirect response model proposed by Jusko et al. ${ }^{15,16)}$ was applied to account for the olanzapine-induced increase in epinephrine secretion and subsequent increase in serum glucose. The rate of change in the serum concentration of epinephrine $\left(C_{\mathrm{Epi}}\right)$ was described by:

$$
\frac{\mathrm{d} C_{\mathrm{Epi}}}{\mathrm{d} t}=k_{\mathrm{in}, \mathrm{Epi}}-\frac{k_{\mathrm{in}, \mathrm{Epi}}}{C_{\mathrm{Epi}(0)}} \cdot C_{\mathrm{Epi}}
$$

where $k_{\mathrm{in}, \mathrm{Epi}}$ and $C_{\mathrm{Epi}(0)}$ represent the zero order constant for epinephrine input and the serum concentration of epinephrine at baseline. The stimulation process of olanzapine on epinephrine input was assumed to be proportional to $C_{1, \mathrm{OLZ}}$. Therefore, the rate of change in $C_{\mathrm{Epi}}$ in the presence of olanzapine was described by:

$$
\frac{\mathrm{d} C_{\mathrm{Epi}}}{\mathrm{d} t}=k_{\mathrm{in}, \mathrm{Epi}} \cdot\left(1+K_{\mathrm{S}, \mathrm{Epi}} \cdot C_{1, \mathrm{OLZ}}\right)-\frac{k_{\mathrm{in}, \mathrm{Epi}}}{C_{\mathrm{Epi}(0)}} \cdot C_{\mathrm{Epi}}
$$

where $K_{\mathrm{S} \text {,Epi }}$ represents the proportionality constant for the stimulation of epinephrine input by olanzapine. The rate of change in the serum concentration of glucose $\left(C_{\mathrm{Glu}}\right)$ was described by:

$$
\frac{\mathrm{d} C_{\mathrm{Glu}}}{\mathrm{d} t}=k_{\mathrm{in}, \mathrm{Glu}}-\frac{k_{\mathrm{in}, \mathrm{Glu}}}{C_{\mathrm{Glu}(0)}} \cdot C_{\mathrm{Glu}}
$$


where $k_{\text {in,Glu }}$ and $C_{\mathrm{Glu}(0)}$ represent the zero order constant for glucose input and the serum concentration of glucose at baseline. By assuming a non-linear stimulation effect of epinephrine on glucose input, the rate of change in $C_{\mathrm{Glu}}$ in the presence of olanzapine was described by:

$$
\begin{aligned}
\frac{\mathrm{d} C_{\mathrm{Glu}}}{\mathrm{d} t}= & k_{\mathrm{in}, \mathrm{Glu}} \cdot\left(1+\frac{E_{\mathrm{max}, \mathrm{Glu}}}{E C_{50, \mathrm{Glu}}+\left(C_{\mathrm{Epi}}-C_{\mathrm{Epi}(0)}\right)}\right. \\
& \left.\times\left(C_{\mathrm{Epi}}-C_{\mathrm{Epi}(0)}\right)\right)-\frac{k_{\mathrm{in}, \mathrm{Glu}}}{C_{\mathrm{Glu}(0)}} \cdot C_{\mathrm{Glu}}
\end{aligned}
$$

where $E_{\text {max,Glu }}$ and $E C_{50, \text { Glu }}$ represent the maximum effect and epinephrine concentration corresponding to $50 \%$ of the maximum effect for the stimulation of glucose input by epinephrine. The time courses of $C_{1, \mathrm{OLZ}}, C_{\mathrm{Epi}}$, and $C_{\mathrm{Glu}}$ for all dose levels were simultaneously fit to the differential Eqs. 1, 2, 4, and 6 by the MULTI (RUNGE) program with the Damping Gauss Newton method algorithm. ${ }^{17)}$ Model selection was based on assessment of graphical diagnostics and comparison of the Akaike's Information Criterion (AIC), provided by MULTI (RUNGE).

Statistical Methods All data represent the mean \pm standard deviation (S.D.). Statistical evaluations were performed using Tukey-Kramer multiple comparison tests. Differences were considered significant at $p<0.05$.

\section{RESULTS}

Effects of the Single Intravenous Administration of Olanzapine on Serum Concentrations of Glucose, Corti-
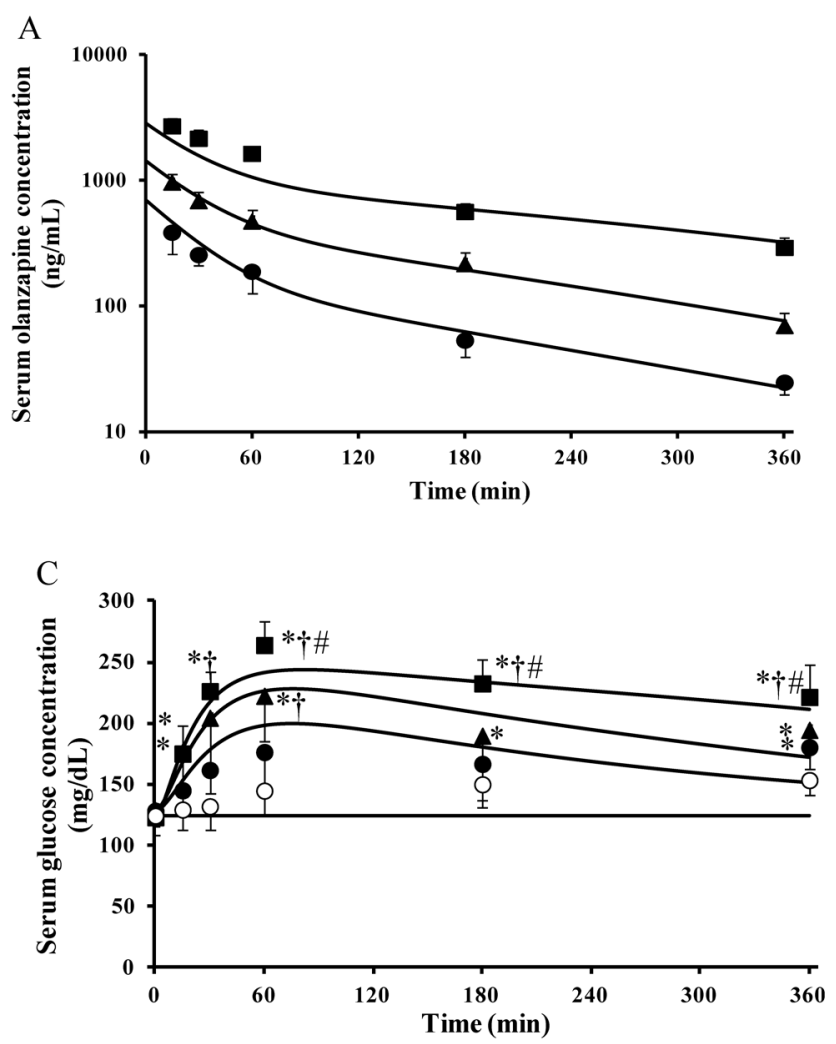

costerone, Glucagon, and Insulin in Rats Serum concentrations of glucose and insulin increased in a dose-dependent manner after the infusion of olanzapine (Figs. 2A, B). Although the serum concentration of corticosterone increased after the administration of olanzapine, no significant difference was observed among the olanzapine dose groups (Fig. 2C). The administration of olanzapine did not affect the serum concentration of glucagon (Fig. 2D).

Effects of the Single Intravenous Administration of Olanzapine on the Serum Concentration of Epinephrine in Rats Serum concentrations of epinephrine (Fig. 3B) and glucose (Fig. 3C) increased in a dose-dependent manner after the infusion of olanzapine.

Effects of the Single Intravenous Administration of Olanzapine on the Serum Concentration of Histamine in Rats The serum concentration of glucose significantly increased $60 \mathrm{~min}$ after the administration of olanzapine (Fig. $4 \mathrm{~A})$. In contrast, the administration of olanzapine did not affect the serum concentration of histamine (Fig. 4B).

Effects of Propranolol on Olanzapine-Induced Increases in the Serum Concentration of Glucose in Rats Olanzapine-induced increases in the serum concentration of glucose were suppressed by the pretreatment with propranolol (Fig. $5 \mathrm{~A})$. The serum concentration of epinephrine increased in the propranolol/olanzapine and saline/olanzapine administration groups (Fig. 5B). The serum concentration of olanzapine was not affected by the propranolol injection (Fig. 5C).

Pharmacokinetic-Pharmacodynamic Modeling of Olanzapine-Induced Hyperglycemia The time courses of serum olanzapine, epinephrine, and glucose for all dose levels were

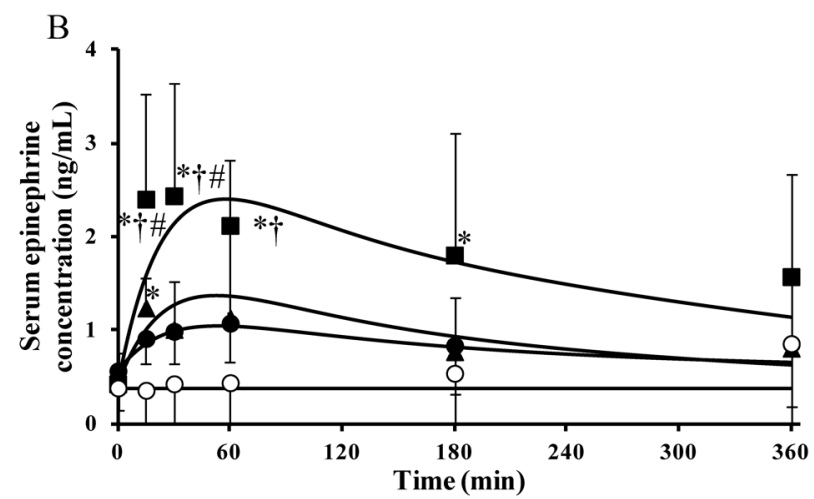

Fig. 3. Serum Concentration-Time Profiles of Olanzapine (A), Epinephrine (B), and Glucose (C) after the Intravenous Infusion of Saline ( $\bigcirc$, as Control, $n=8)$, Olanzapine at $2.5 \mathrm{mg} / \mathrm{kg}(\boldsymbol{O}, n=8)$, Olanzapine at $5 \mathrm{mg} / \mathrm{kg}(\boldsymbol{\Lambda}, n=7)$, or Olanzapine at $10 \mathrm{mg} / \mathrm{kg}(\boldsymbol{\square}, n=7)$ in $\mathrm{Rats}$

Each point represents the mean \pm S.D. ${ }^{*} p<0.05 v s$. the control group, ${ }^{\dagger} p<0.05 v s$. olanzapine $2.5 \mathrm{mg} / \mathrm{kg}$ and ${ }^{\#} p<0.05 v s$. olanzapine $5 \mathrm{mg} / \mathrm{kg}$ (the Tukey-Kramer test) Solid lines denote computer-fitted curves. 
A

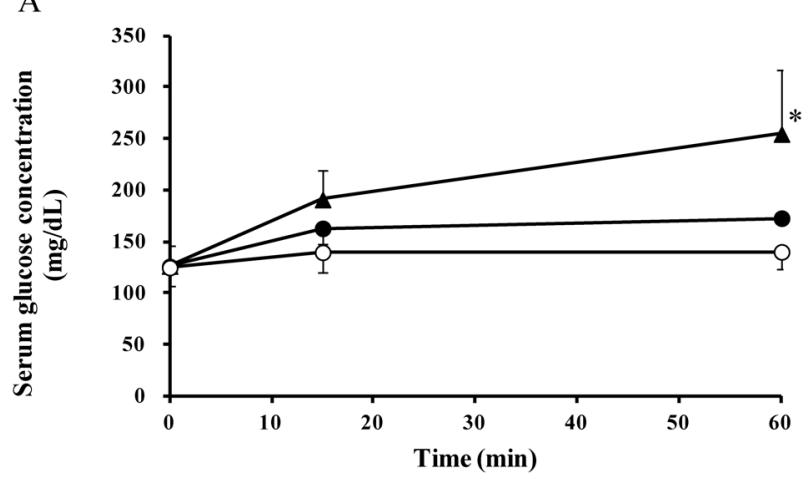

B

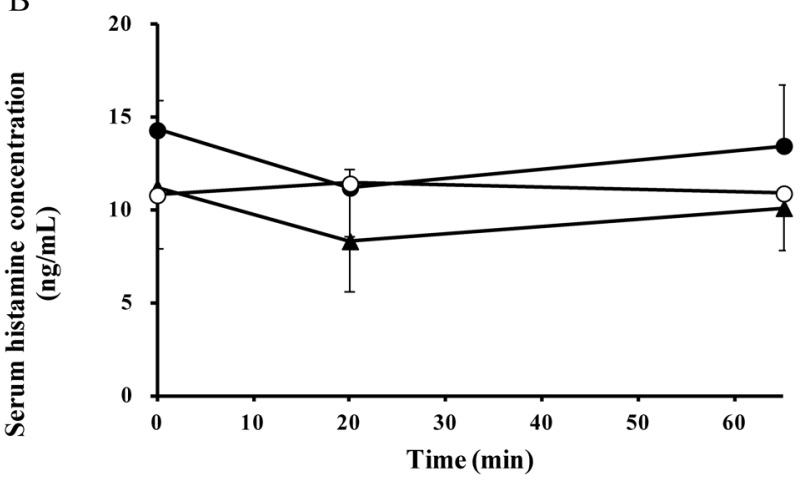

Fig. 4. Serum Concentration-Time Profiles of Glucose (A) and Histamine (B) after the Intravenous Infusion of Saline $(\bigcirc$, as Control, $n=3)$, Olanzapine at $2.5 \mathrm{mg} / \mathrm{kg}(\boldsymbol{\bullet}, n=3)$, or Olanzapine at $5 \mathrm{mg} / \mathrm{kg}(\boldsymbol{\Lambda}, n=3)$ in Rats

Each point represents the mean \pm S.D. ${ }^{*} p<0.05 v$ s. the control group (the Tukey-Kramer test).
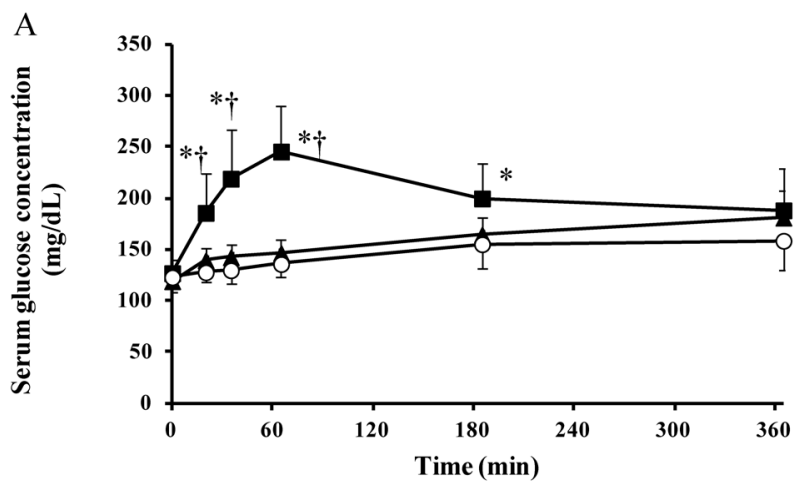

C

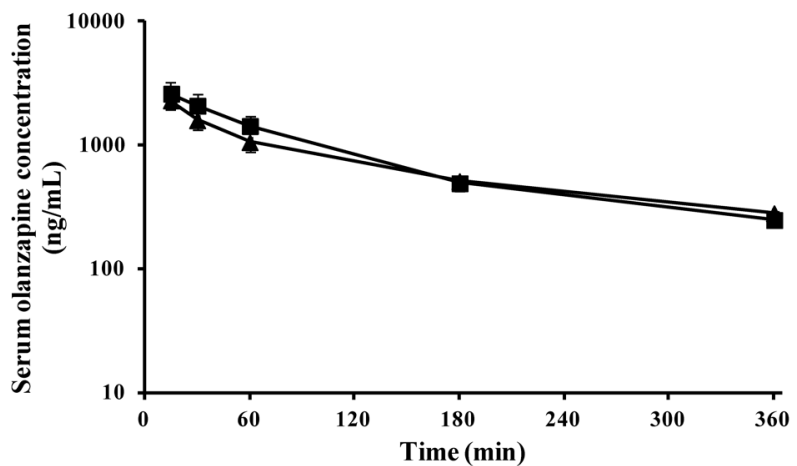

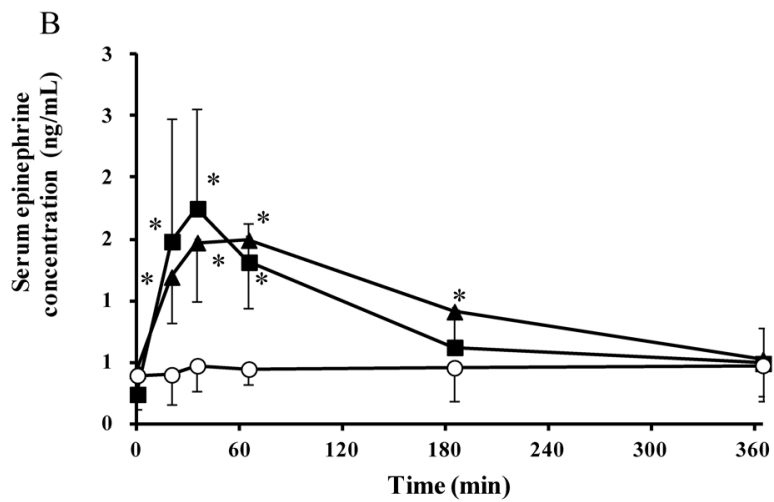

Fig. 5. Effects of Propranolol (2 mg/kg) on Serum Concentrations of Glucose (A), Epinephrine (B), and Olanzapine (C) after the Intravenous Infusion of Olanzapine $(10 \mathrm{mg} / \mathrm{kg})$

$\bigcirc$ : Rats treated with propranolol and saline $(n=8), \mathbf{\square}$ : rats treated with saline and olanzapine $(n=8), \boldsymbol{\Delta}$ : rats treated with propranolol and olanzapine $(n=8)$. Each point represents the mean \pm S.D. ${ }^{*} p<0.05 v s$. propranolol/saline, ${ }^{\dagger} p<0.05 v s$. propranolol/olanzapine (the Tukey-Kramer test).

Table 1. Pharmacokinetic and Pharmacodynamic Parameters of Olanzapine-Induced Hyperglycemia after Its Intravenous Administration to Rats

\begin{tabular}{lclc}
\hline \hline Pharmacokinetic parameters & \multicolumn{3}{c}{ Pharmacodynamic parameters } \\
\hline$k_{12}(/ \mathrm{min})$ & $0.0207 \pm 0.0068$ & $k_{\text {in, Epi }}(\mu \mathrm{g} / \mathrm{L} / \mathrm{min})$ & $0.00864 \pm 0.00192$ \\
$k_{21}(/ \mathrm{min})$ & $0.0118 \pm 0.0029$ & $K_{\mathrm{S}, \mathrm{Epi}}(\mathrm{L} / \mu \mathrm{g})$ & $0.00424 \pm 0.0049$ \\
$V_{1}(\mathrm{~L} / \mathrm{kg})$ & $3.52 \pm 0.57$ & $k_{\mathrm{in}, \mathrm{Glu}}(\mathrm{mg} / \mathrm{dL} / \mathrm{min})$ & $8.60 \pm 4.89$ \\
$V_{\text {max }, \mathrm{OLZ}}(\mu \mathrm{g} / \mathrm{min} / \mathrm{kg})$ & $24.6 \pm 6.3$ & $E_{\text {max }, \mathrm{Glu}}$ & $1.28 \pm 0.51$ \\
$K_{\mathrm{m}, \mathrm{OLZ}}(\mu \mathrm{g} / \mathrm{L})$ & $273 \pm 117$ & $E C_{50, \mathrm{Glu}}(\mu \mathrm{g} / \mathrm{L})$ & $0.594 \pm 0.502$ \\
\hline
\end{tabular}

Data represent the mean \pm S.D. $k_{12}$, the first order transfer rate constant for a drug moving from the central to the peripheral compartment; $k_{21}$, the first order transfer rate constant for a drug moving from the peripheral to the central compartment; $V_{1}$, the distribution volume of olanzapine in the central compartment; $V_{\text {max,oLz }}$, the maximum velocity of the elimination of olanzapine; $K_{\mathrm{m}, \mathrm{OLZ}}$, the Michaelis constant for the elimination of olanzapine; $k_{\mathrm{in}, \mathrm{Ep}}$, the zero order constant for epinephrine input; $K_{\mathrm{S}, \text { Ep }}$, the proportionality constant for the stimulation of epinephrine input by olanzapine; $k_{\text {in,Glu }}$, the zero order constant for glucose input; $E_{\text {max,Glu }}$ the maximum effect for the stimulation of glucose input by epinephrine; $E C_{50, \text { Glu }}$, the concentration of epinephrine corresponding to $50 \%$ of the maximum effect for the stimulation of glucose input by epinephrine. 
simultaneously fit to the differential Eqs. 1, 2, 4, and 6. The fitted curves for mean serum olanzapine, epinephrine, and glucose concentrations following the administration of olanzapine were a good match to the data obtained (Fig. 3), and serum glucose concentrations were described well by our developed model (Fig. 1). The pharmacokinetic and pharmacodynamic parameters estimated are shown in Table 1.

\section{DISCUSSION}

In order to develop an experimental animal model of olanzapine-induced hyperglycemia, a high dose of olanzapine was administered intravenously to rats. In the present study, the serum concentrations of glucose and insulin increased in a dose-dependent manner after the single intravenous infusion of olanzapine (Figs. 2A, B). These results suggest that rats are appropriate experimental animals for investigating olanzapine-induced acute hyperglycemia in humans, and also that the secretion of insulin with a high glucose stimulation is not inhibited by the administration of olanzapine.

Many endogenous compounds, such as catecholamines, glucagon, and glucocorticoids, are recognized as factors that regulate the serum concentration of glucose. ${ }^{18)}$ In an attempt to clarify the mechanism underlying olanzapine-induced acute hyperglycemia, we investigated the effects of the single intravenous administration of olanzapine on the serum concentrations of several endogenous substances.

Epinephrine activates glycogenolysis and glyconeogenesis in order to elevate serum glucose levels via the stimulation of $\beta_{2}$-adrenergic receptors. ${ }^{19-21)}$ Our results showed that the serum concentration of epinephrine increased in a dosedependent manner after the administration of olanzapine (Fig. 3B). Furthermore, the pretreatment with propranolol ( $\beta$-adrenergic antagonist) completely suppressed olanzapineinduced elevations in serum glucose levels without affecting the concentrations of serum olanzapine and epinephrine (Fig. 5). Our developed pharmacokinetic-pharmacodynamic model, which assumes that the olanzapine-induced secretion of epinephrine leads to elevated serum glucose concentrations (Fig. 1), also appeared to satisfactorily characterize olanzapineinduced hyperglycemia (Fig. 3). These results suggest that epinephrine is involved in elevating serum glucose levels with the single administration of olanzapine.

We previously reported that novel fluoroquinolone antimicrobial agents such as gatifloxacin, levofloxacin, and moxifloxacin increased serum glucose concentrations, and this was associated with the induction of histamine release by these drugs, leading to elevations in serum epinephrine concentrations. ${ }^{12,22-24)}$ On the other hand, the single intravenous injection of olanzapine did not affect the serum concentration of histamine (Fig. 4B). These results suggest that the mechanism responsible for olanzapine-induced elevations in serum epinephrine levels differs from that used by fluoroquinolone antimicrobial agents.

Glucocorticoids induce hyperglycemia through gluconeogenesis by increasing the rate of transcription of genes that encode gluconeogenic enzymes such as phosphoenolpyruvate carboxykinase and glucose-6-phosphatase. ${ }^{25-27)}$ In addition, glucocorticoids induce peripheral insulin resistance. ${ }^{27)}$ Although the serum concentration of corticosterone increased after the infusion of olanzapine in the present study, no signif- icant difference was noted among the olanzapine dose groups (Fig. 2C). Assié et al. also reported that olanzapine increased plasma glucose and corticosterone levels in rats. ${ }^{28)}$ However, in the olanzapine dose range of $10-40 \mathrm{mg} / \mathrm{kg}$ per os (p.o.), plasma glucose levels increased with elevations in the dose of olanzapine administered, even though plasma corticosterone levels remained unchanged. These results suggest that corticosterone is not as strongly associated with olanzapineinduced elevations in serum glucose levels.

Glucagon plays a critical role in maintaining glucose homeostasis. In order to increase blood glucose levels, glucagon enhances hepatic glucose output by promoting glycogenolysis and gluconeogenesis and suppressing glycogenesis and glycolysis. ${ }^{29)}$ Smith et al. previously reported that glucagon levels were higher in rats following a clozapine (structurally similar to olanzapine) injection, and that this increase was the major contributor to elevated blood glucose levels. ${ }^{30)}$ In contrast, the administration of olanzapine did not affect the serum concentration of glucagon (Fig. 2D). These results suggest that glucagon is not associated with olanzapine-induced elevations in blood glucose levels, and the mechanism responsible for the induction of hyperglycemia with a single administration of olanzapine may differ from that by clozapine. Olanzapine exhibited a rich receptor binding profile, resembling that of clozapine; however, the affinity of olanzapine for several receptors differed from that of clozapine. ${ }^{31)}$ This difference may be associated with the different mechanisms responsible for drug-induced hyperglycemia.

Ikegami et al. recently reported that olanzapine activated AMPK in the hypothalamus, and, as a consequence, increased hepatic glucose production via the sympathetic nervous system. ${ }^{8)}$ The sympathetic nervous system promotes hepatic glucose production by direct sympathetic innervations of the liver, adrenal medullary epinephrine, and pancreatic glucagon. $^{32)}$ Our results show that the pathway of epinephrine release is mainly responsible for olanzapine-induced hyperglycemia under fasted conditions, while the pathway of glucagon release is not. On the other hand, the relative importance of these pathways may be changed by a number of factors including environment, species, diet, and alterations in neurotransmission. ${ }^{32)}$ Further investigations are needed in order to clarify the mechanism responsible for olanzapine-induced hyperglycemia.

The physiological glucose regulatory system is complex. Previous studies used a dual indirect response model involving feedback regulation and homeostasis to describe the glucose-insulin system. ${ }^{33,34)}$ In contrast, in order to elucidate the mechanism underlying olanzapine-induced hyperglycemia, we developed a simple pharmacokinetic-pharmacodynamic model assuming that the olanzapine-induced secretion of epinephrine increases the serum concentration of glucose (Fig. 1). In this model, the $k_{\mathrm{in}, \mathrm{Glu}} / C_{\mathrm{Glu}(0)}$ (the first-order rate constant for the elimination of glucose) is assumed to contain the insulin effect decreasing glucose concentrations. In a preliminary study, we compared the two structural models (linear and $E_{\max }$ model) to describe the relationship between $C_{\text {Epi }}$ and its stimulating effect on glucose input. The $E_{\max }$ model (Eq. 6) was adopted based on the lower value of AIC. We showed that our developed model successfully described the time courses of olanzapine, epinephrine, and glucose concentrations (Fig. 3). Furthermore, the estimated glucose half-life 
(approximately $10.2 \mathrm{~min}$ ) calculated by the $k_{\mathrm{in}, \mathrm{Glu}} / C_{\mathrm{Glu}(0)}$ value in the present study was similar to previously reported values (approximately $15.6-43.8 \mathrm{~min})^{33-35)}$ Thus, under conditions in which the glucose-induced secretion of insulin and effects of insulin on glucose utilization are not impaired, a simple model such as ours appears to be sufficient to describe the kinetics of glucose.

In the present study, a high dose of olanzapine $(2.5-10 \mathrm{mg} /$ $\mathrm{kg}$ ) was administered to rats and the serum concentrations of olanzapine $15 \mathrm{~min}$ after olanzapine administration ranged from 394 to $2763 \mathrm{ng} / \mathrm{mL}$ depending on the dose. In contrast, a clinical daily dose of olanzapine is $5-20 \mathrm{mg} / \mathrm{d}$ and the therapeutic range of olanzapine is $20-50 \mathrm{ng} / \mathrm{mL}$. $^{36)}$ Thus, the serum concentrations of olanzapine in the present study were higher than those at clinical doses of olanzapine. Though acute hyperglycemias were reported in patients treated with $2.5-10 \mathrm{mg} / \mathrm{d}$ of olanzapine, ${ }^{6,7)}$ it is still unknown about the concentrations of olanzapine at the onset of acute hyperglycemia in clinical setting. Therefore, the concentration-effect relationship of olanzapine-induced acute hyperglycemia in humans remains to be solved.

In conclusion, a single intravenous dose of olanzapine increased the serum concentration of glucose in a dosedependent manner in rats, and epinephrine is involved in the process of olanzapine-induced acute hyperglycemia.

Acknowledgments This work was supported by a Grantin-Aid for Scientific Research (24590180) from the Japan Society for the Promotion of Science, and by a Grant from the Research Foundation for Pharmaceutical Sciences.

Conflict of Interest The authors declare no conflict of interest.

\section{REFERENCES}

1) Bymaster FP, Calligaro DO, Falcone JF, Marsh RD, Moore NA, Tye NC, Seeman P, Wong DT. Radioreceptor binding profile of the atypical antipsychotic olanzapine. Neuropsychopharmacology, 14, 87-96 (1996)

2) Zhang JP, Gallego JA, Robinson DG, Malhotra AK, Kane JM, Correll CU. Efficacy and safety of individual second-generation $v s$. first-generation antipsychotics in first-episode psychosis: a systematic review and meta-analysis. Int. J. Neuropsychopharmacol., 16 , 1205-1218 (2013).

3) Seaburg HL, McLendon BM, Doraiswamy PM. Olanzapine-associated severe hyperglycemia, ketonuria, and acidosis: case report and review of literature. Pharmacotherapy, 21, 1448-1454 (2001).

4) Deng C. Effects of antipsychotic medications on appetite, weight, and insulin resistance. Endocrinol. Metab. Clin. North Am., 42, 545-563 (2013)

5) Teff KL, Rickels MR, Grudziak J, Fuller C, Nguyen HL, Rickels K. Antipsychotic-induced insulin resistance and postprandial hormonal dysregulation independent of weight gain or psychiatric disease. Diabetes, 62, 3232-3240 (2013).

6) Kohen I, Gampel M, Reddy L, Manu P. Rapidly developing hyperglycemia during treatment with olanzapine. Ann. Pharmacother., 42, 588-591 (2008).

7) Mizukami T, Kimura M, Yamamoto T, Maruyama K. First administration of olanzapine caused acute onset hyperglycemia on a bipolar-I patient. Jpn. J. Clin. Psycopharmacol., 8, 1751-1754 (2005).

8) Ikegami M, Ikeda H, Ohashi T, Ohsawa M, Ishikawa Y, Kai M, Kamei A, Kamei J. Olanzapine increases hepatic glucose produc- tion through the activation of hypothalamic adenosine 5 '-monophosphate-activated protein kinase. Diabetes Obes. Metab., 15, $1128-1135$ (2013).

9) Ikegami M, Ikeda H, Ohashi T, Kai M, Osada M, Kamei A, Kamei J. Olanzapine-induced hyperglycemia: possible involvement of histaminergic, dopaminergic and adrenergic functions in the central nervous system. Neuroendocrinology, 98, 224-232 (2013).

10) Ling S, Jamali F. Effect of cannulation surgery and restraint stress on the plasma corticosterone concentration in the rat: application of an improved corticosterone HPLC assay. J. Pharm. Pharm. Sci., 6, 246-251 (2003)

11) Zhang G, Terry AV Jr, Bartlett MG. Simultaneous determination of five antipsychotic drugs in rat plasma by high performance liquid chromatography with ultraviolet detection. J. Chromatogr. B Analyt. Technol. Biomed. Life Sci., 856, 20-28 (2007).

12) Ishiwata Y, Sanada Y, Yasuhara M. Effects of gatifloxacin on serum glucose concentration in normal and diabetic rats. Biol. Pharm. Bull., 29, 527-531 (2006).

13) Furuhata K, Hayakawa $H$, Soumi K, Arai H, Watanabe $Y$, Narita H. Histamine-releasing properties of T-3762, a novel fluoroquinolone antimicrobial agent in intravenous use. I. Effects of doses and infusion rate on blood pressure, heart rate and plasma histamine concentration. Biol. Pharm. Bull., 21, 456-460 (1998).

14) Aravagiri M, Teper Y, Marder SR. Pharmacokinetics and tissue distribution of olanzapine in rats. Biopharm. Drug Dispos., 20, 369-377 (1999).

15) Dayneka NL, Garg V, Jusko WJ. Comparison of four basic models of indirect pharmacodynamic responses. J. Pharmacokinet. Biopharm., 21, 457-478 (1993).

16) Jusko WJ, Ko HC. Physiologic indirect response models characterize diverse types of pharmacodynamic effects. Clin. Pharmacol. Ther., 56, 406-419 (1994).

17) Yamaoka K, Nakagawa T. A nonlinear least squares program based on differential equations, MULTI (RUNGE), for microcomputers. $J$. Pharmacobiodyn., 6, 595-606 (1983).

18) Shamoon H, Hendler R, Sherwin RS. Synergistic interactions among antiinsulin hormones in the pathogenesis of stress hyperglycemia in humans. J. Clin. Endocrinol. Metab., 52, 1235-1241 (1981).

19) Cherrington AD, Fuchs H, Stevenson RW, Williams PE, Alberti $\mathrm{KG}$, Steiner KE. Effect of epinephrine on glycogenolysis and gluconeogenesis in conscious overnight-fasted dogs. Am. J. Physiol., 247, E137-E144 (1984).

20) Kawai Y, Arinze IJ. $\beta$-Adrenergic receptors in rabbit liver plasma membranes. Predominance of $\beta 2$-receptors and mediation of adrenergic regulation of hepatic glycogenolysis. J. Biol. Chem., 258, 4364-4371 (1983).

21) Kim K, Cho SC, Cova A, Jang IS, Park SC. Alterations of epinephrine-induced gluconeogenesis in aging. Exp. Mol. Med., 41, 334-340 (2009)

22) Ishiwata Y, Itoga $Y$, Yasuhara M. Effect of levofloxacin on serum glucose concentration in rats. Eur. J. Pharmacol., 551, 168-174 (2006).

23) Ishiwata Y, Yasuhara M. Gatifloxacin-induced histamine release and hyperglycemia in rats. Eur. J. Pharmacol., 645, 192-197 (2010).

24) Ishiwata Y, Takahashi Y, Nagata M, Yasuhara M. Effects of moxifloxacin on serum glucose concentrations in rats. Biol. Pharm. Bull., 36, 686-690 (2013)

25) Granner D, Pilkis S. The genes of hepatic glucose metabolism. $J$. Biol. Chem., 265, 10173-10176 (1990).

26) van Schaftingen E, Gerin I. The glucose-6-phosphatase system. Biochem. J., 362, 513-532 (2002).

27) Rafacho A, Ortsater H, Nadal A, Quesada I. Glucocorticoid treatment and endocrine pancreas function: implications for glucose homeostasis, insulin resistance and diabetes. J. Endocrinol., 223, R49-R62 (2014). 
28) Assié MB, Carilla-Durand E, Bardin L, Maraval M, Aliaga M, Malfétes N, Barbara M, Newman-Tancredi A. The antipsychotics clozapine and olanzapine increase plasma glucose and corticosterone levels in rats: comparison with aripiprazole, ziprasidone, bifeprunox and F15063. Eur. J. Pharmacol., 592, 160-166 (2008).

29) Jiang G, Zhang BB. Glucagon and regulation of glucose metabolism. Am. J. Physiol. Endocrinol. Metab., 284, E671-E678 (2003).

30) Smith GC, Chaussade C, Vickers M, Jensen J, Shepherd PR. Atypical antipsychotic drugs induce derangements in glucose homeostasis by acutely increasing glucagon secretion and hepatic glucose output in the rat. Diabetologia, 51, 2309-2317 (2008).

31) Zhang W, Bymaster FP. The in vivo effects of olanzapine and other antipsychotic agents on receptor occupancy and antagonism of dopamine D1, D2, D3, 5HT2A and muscarinic receptors. Psychopharmacology, 141, 267-278 (1999).
32) Nonogaki K. New insights into sympathetic regulation of glucose and fat metabolism. Diabetologia, 43, 533-549 (2000).

33) Sato S, Katayama K, Kakemi M, Koizumi T. A kinetic study of chlorpromazine on the hyperglycemic response in rats. II. Effect of chlorpromazine on plasma glucose. J. Pharmacobiodyn., 11, 492-503 (1988)

34) Cao Y, Gao W, Jusko WJ. Pharmacokinetic/pharmacodynamic modeling of GLP-1 in healthy rats. Pharm. Res., 29, 1078-1086 (2012).

35) Takahashi Y, Ishiwata Y, Kojima Y, Yasuhara M. Pharmacodynamics of cibenzoline-induced hypoglycemia in rats. Drug Metab. Pharmacokinet., 26, 242-247 (2011).

36) Mauri MC, Paletta S, Maffini M, Colasanti A, Dragogna F, Di Pace C, Altamura AC. Clinical pharmacology of atypical antipsychotics: an update. EXCLI J., 13, 1163-1191 (2014). 